Meta

Journal des traducteurs

Translators' Journal

\title{
L'apport de la psychologie cognitive à la didactique de la traduction
}

\section{Michel Politis}

Volume 52, numéro 1, mars 2007

Traductologie : une science cognitive

URI : https://id.erudit.org/iderudit/014730ar

DOI : https://doi.org/10.7202/014730ar

Aller au sommaire du numéro

Éditeur(s)

Les Presses de l'Université de Montréal

ISSN

0026-0452 (imprimé)

1492-1421 (numérique)

Découvrir la revue

Citer cet article

Politis, M. (2007). L'apport de la psychologie cognitive à la didactique de la traduction. Meta, 52(1), 156-163. https://doi.org/10.7202/014730ar
Résumé de l'article

L'étude des processus cognitifs durant l'accomplissement de l'acte traductionnel constitue un défi important pour les spécialistes en didactique de la traduction. Selon toutes les indications, la connaissance de ces processus peut constituer le fondement pour esquisser des nouvelles méthodes d'enseignement qui viseront au développement des aptitudes cognitives des traducteurs en vue d'améliorer leurs performances qualitatives et quantitatives. Les recherches en la matière doivent porter sur deux axes principaux : celui de la lecture, en tant qu'activité cognitive qui permet au traducteur d'aborder le contenu du texte qu'il a à traduire et de vérifier par la suite le résultat de son travail, ainsi que celui du fonctionnement du système mnésique lors de l'acte traductionnel. À titre indicatif les recherches peuvent viser à la formation de la représentation mentale du texte à traduire et son traitement lors de la traduction, l'étude des stratégies en vue d'accomplir l'acte traductionnel, l'étude des stratégies de gestion des ressources cognitives du traducteur, etc
Ce document est protégé par la loi sur le droit d'auteur. L'utilisation des services d’Érudit (y compris la reproduction) est assujettie à sa politique d'utilisation que vous pouvez consulter en ligne.

https://apropos.erudit.org/fr/usagers/politique-dutilisation/ 


\title{
L'apport de la psychologie cognitive à la didactique de la traduction
}

\author{
MICHEL POLITIS \\ Université ionienne, Corfou, Grèce \\ politis@dfli.ionio.gr
}

\begin{abstract}
RÉSUMÉ
L'étude des processus cognitifs durant l'accomplissement de l'acte traductionnel constitue un défi important pour les spécialistes en didactique de la traduction. Selon toutes les indications, la connaissance de ces processus peut constituer le fondement pour esquisser des nouvelles méthodes d'enseignement qui viseront au développement des aptitudes cognitives des traducteurs en vue d'améliorer leurs performances qualitatives et quantitatives. Les recherches en la matière doivent porter sur deux axes principaux: celui de la lecture, en tant qu'activité cognitive qui permet au traducteur d'aborder le contenu du texte qu'il a à traduire et de vérifier par la suite le résultat de son travail, ainsi que celui du fonctionnement du système mnésique lors de l'acte traductionnel. À titre indicatif les recherches peuvent viser à la formation de la représentation mentale du texte à traduire et son traitement lors de la traduction, l'étude des stratégies en vue d'accomplir l'acte traductionnel, l'étude des stratégies de gestion des ressources cognitives du traducteur, etc.
\end{abstract}

\begin{abstract}
The study of the mental processes taking place when translating is an important challenge for specialists of translation didactics. Based on all indications, knowing all these processes may be the basis for designing new teaching methods that aim to develop the translators' skills and to improve their quantitative and qualitative performance. Research in this field should focus mainly on two axes: $i$. the reading phase as a cognitive activity through which the translator gains access to the content of the text to be translated and thus controls the outcome of his/her effort; and ii. the function of the mnemonic system during the translation act. Indicative topics for research could be the way the text for translation is mentally represented, as well as the way this representation is processed when translating. It would also be useful to study the strategies employed for translation performance, the translator's strategies for managing his/her cognitive resources, etc.
\end{abstract}

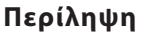

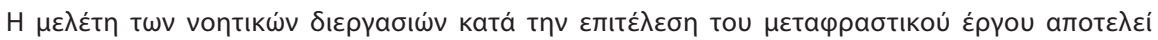

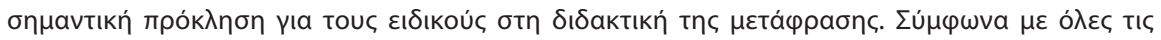

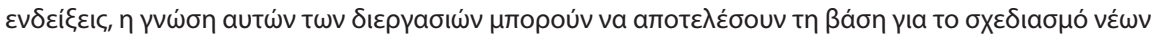

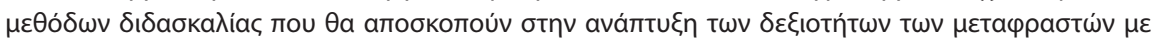

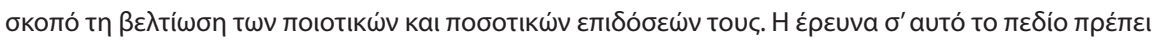

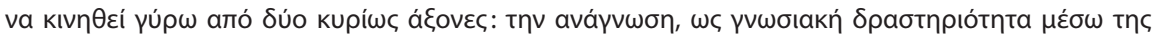

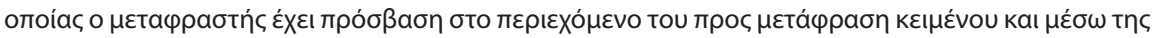

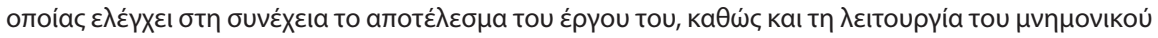

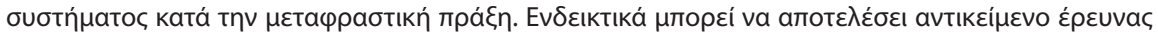

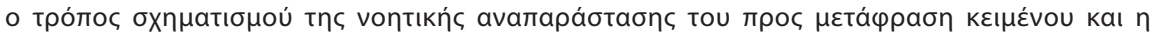

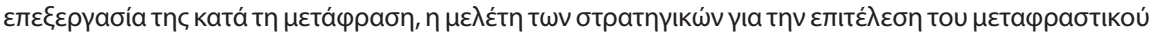

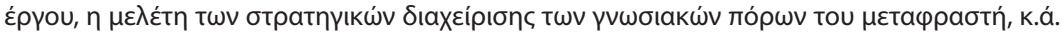

\section{MOTS-CLÉS/KEYWORDS}

psychologie cognitive, aptitudes cognitives des traducteurs, didactique de la traduction 
Depuis quelques décennies, particulièrement depuis le colloque sur La compréhension du langage qui s'est tenu à Créteil en septembre 1980 (ESIT 1980), la psychologie cognitive apporte son savoir à la traductologie. Jusqu'alors la psychologie n'offrait pas les outils nécessaires pour étudier le processus de la traduction car l'approche behaviouriste, en vogue à l'époque, excluait de ses préoccupations toute étude de ce qui n'était pas scientifiquement observable. Les premières approches que nous pourrions considérer comme «cognitivistes» étaient celles inspirées de la théorie de la communication, de la cybernétique et de l'intelligence artificielle qui ont été conçues en vue d'être exploitées dans le cadre des projets de recherche en traduction automatique ou en TAO. Ce tournant vers la psychologie cognitive est le résultat de la prise de conscience du fait que les recherches dans le domaine de la traduction automatique ne pouvaient pas offrir une solution fiable aux besoins du marché de la traduction. Constatant cette impasse, un nombre croissant de traductologues a commencé à formuler des hypothèses selon lesquelles si nous arrivons à mieux connaître le mode de fonctionnement du cerveau du traducteur nous serons en mesure d'élaborer des cursus spécifiques permettant la formation de traducteurs plus performants et plus fiables. Au début, cet apport n'était que relatif, mais grâce au développement spectaculaire des sciences cognitives ces dernières années et à l'intensification des rapports entre traductologues et psychologues, nous assistons à une prise de conscience croissante du caractère cognitif de l'acte traductionnel. L'introduction de paramètres des sciences cognitives s'est effectuée dans un premier temps principalement par l'École interprétative qui considère le traducteur/interprète comme une entité cognitive qui a pour mission de faciliter la communication.

Une des premières tentatives expérimentales pour étudier le processus de la traduction a été celle qui consistait à appliquer la méthode du Think Aloud Protocol (TAP). L'expérience de Maurice Perginer (1980: 425-458) a été suivie par d'autres, telles celles de Wolfgang Lörscher, de Hans-Peter Krings, de Julianne House, de Donald Charles Kiraly et de Paul Kussmaul. Cette méthode, bien qu'elle ait connu plusieurs critiques pour ses défaillances méthodologiques, a contribué à la prise de conscience que la «boîte-noire» des behaviouristes pouvait être ouverte et analysée. Nous devons également noter que certaines conclusions de ces recherches continuent à inspirer plusieurs formateurs de traducteurs. La principale critique formulée à l'égard de cette méthode est qu'elle n'implique pas nécessairement l'extériorisation de tout ce qui se passe dans le cerveau humain. Ceci résulte du fait que certains processus cognitifs ne sont pas perceptibles par l'introspection ou parce que entre le cerveau du traducteur et la description de ce qui s'y passe s'intercalent plusieurs «filtres», ce qui a comme conséquence la déformation de ce qui s'extériorise.

Malgré les difficultés et les contraintes d'ordre méthodologiques, plusieurs enseignants de traduction étudient les résultats des recherches en psychologie cognitive et intègrent à leurs recherches ainsi qu'à leur enseignement la dimension cognitive. Faisant partie de ce courant, nous avons introduit à nos cours d'initiation à la traduction et de traduction économique, juridique et politique cette dimension. Dans notre communication nous avons l'intention de présenter quelques réflexions concernant certains domaines de la didactique de la traduction qui, selon nous, pourraient faire l'objet de projets de recherche pluridisciplinaires. Nous devons auparavant clarifier certaines notions clés de notre exposé, le champ de notre exposé. Lors des cours de traduction nous nous adressons à un public, à des apprentis traducteurs à qui nous 
transmettons un savoir sous forme de notions de traductologie et un savoir-faire. Or, existe-t-il un apprenti traducteur type à qui nous inculquons les notions de traductologie et à qui nous lui transmettons notre savoir-faire? Certainement pas, car les systèmes de sélection appliqués dans les différents pays ne permettent pas de minimiser les écarts au niveau des compétences afin d'aboutir à des auditoires plus ou moins homogènes. Étant donné que les systèmes de sélection varient selon les pays et même selon les universités, nous devons être prudents lors de la formulation d'hypothèses ou d'affirmations dans le domaine de la didactique de la traduction. Si l'apprenti traducteur type ou l'auditoire d'apprentis traducteurs types n'existent pas, faut-il se pencher sur eux pour mieux les connaître en tant qu'entités cognitives? À notre avis, la réponse doit être à priori positive dans la mesure où la connaissance des qualités cognitives des apprentis traducteurs nous permet de mieux les comprendre et ainsi d'organiser un enseignement plus personnalisé. Nous devons, cependant, tenir compte non seulement des limites de la psychologie cognitive, mais aussi des contraintes imposées par le caractère collectif de l'enseignement. La psychologie cognitive peut donc apporter un soutien certain à l'enseignant de la traduction, comme c'est le cas pour la didactique de toutes les matières enseignées au primaire, au secondaire ou au niveau universitaire.

Cet apport peut porter sur deux axes: celui du développement des aptitudes cognitives des apprentis traducteurs et celui de leur prise de conscience de la dimension métacognitive du processus traductionnel. Le développement des aptitudes cognitives des apprentis traducteurs peut s'effectuer en enrichissant les cours de traduction avec des exercices spécifiques en fonction de leur niveau et en fonction d'objectifs précis d'ordre quantitatif ou qualitatif. La prise de conscience de la dimension métacognitive du processus traductionnel peut se réaliser dans le cadre d'un cours de psychologie cognitive et par des références spécifiques lors des cours de traduction. Elle peut consister, d'une part, à aider les apprentis à prendre conscience des aptitudes, des stratégies et des ressources nécessaires pour la réalisation d'une traduction, et, d'autre part, à les aider à prendre conscience des mécanismes cognitifs leur permettant de réguler, de contrôler, de coordonner leur comportement cognitif lors de l'acte traductionnel.

Étant donné que le champ est encore peu exploré, nous devons soutenir des projets de recherche qui auront comme objet d'étude le processus même de la traduction, en le considérant à la fois comme un processus cognitif mais aussi comme une «situation-problème» qui doit être résolue en adoptant une stratégie de résolution et des choix ponctuels. Il s'agit donc d'un processus qui requiert des prises de décisions en fonction des éléments contenus explicitement ou implicitement dans le texte à traduire, de la situation de communication dans laquelle s'insère l'acte traductionnel, et du bagage cognitif des traducteurs. L'approche envisagée ne peut être que pluridisciplinaire, car elle suppose la collaboration de spécialistes en traductologie, en psychologie cognitive, en neuropsychologie, etc. Ces recherches pourraient conduire à une éventuelle reconsidération des cursus des écoles de traduction ainsi que des méthodes d'enseignement de cette discipline en vue d'améliorer la fiabilité et la performance cognitives des traducteurs.

Une activité cognitive qui s'avère très importante lors de la traduction, tant au niveau de la saisie du sens qu'au niveau du contrôle du texte produit par le traducteur, est celle de la lecture. Apparemment, étant donné que les objectifs de ces deux types de lectures divergent, le traducteur adopte des stratégies de lecture différentes. L'étude 
de ces stratégies est un domaine qui pourrait fournir des informations très intéressantes, surtout pour les formateurs de traducteurs. Les recherches dans ce domaine doivent envisager la lecture à la fois comme une activité oculomotrice et une activité cognitive, dans la mesure où il est généralement admis que les mouvements oculaires d'une personne dans une tâche visuelle reflètent en grande partie les processus cognitifs mis en œuvre durant cette activité, et comme une activité cognitive, dans la mesure où elle constitue le moyen par lequel le lecteur perçoit les informations contenues dans le texte qu'il traitera au sein de son système mnésique.

En tant qu'activité oculomotrice la lecture est intéressante car, comme Alexandra Kosma l'a démontré (Kosma 2005), le lecteur-traducteur adopte des stratégies de lecture différentes par rapport au simple lecteur, car, selon toutes les apparences, il adopte des stratégies qui vont lui permettre de préparer la reformulation du sens de l'énoncé. Selon Kosma, ces stratégies se différencient en fonction du type de texte à traduire, de l'expertise du traducteur, etc. Ainsi, elle remarque que les sujets qui font une traduction dite «mot à mot» en ne cherchant que des correspondances de mots et de sens, ont un processus oculaire quasi linéaire, alors que les sujets qui optent pour une stratégie plus synthétique et appliquent l'approche proposée par la théorie interprétative ont un parcours plus exploratoire. Si les stratégies de lecture se différencient donc en fonction de ces paramètres, pouvons-nous formuler certaines conclusions, et à partir de celles-ci, proposer des exercices qui accroîtront les performances des apprentis traducteurs sans porter préjudice à la qualité du sens saisi?

Il nous semble que la psychologie cognitive peut apporter également son savoir dans une étape bien particulière de la traduction, à savoir celle de la lecture du texte produit par le traducteur en vue de vérifier ses choix traductionnels. Lorsque nous lisons une traduction réalisée par un tiers, il est facile d'adopter une attitude neutre et objective à l'égard de cette œuvre, mais lorsque nous sommes face à notre propre traduction, la distanciation par rapport à elle n'est pas évidente. Ceci est beaucoup plus difficile pour l'apprenti traducteur car ses interprétations antérieures, mêmes si elles sont fausses, continuent à être présentes dans sa conscience ou dans son inconscient et interfèrent à chaque tentative d'une nouvelle interprétation. Pour arriver à cette distanciation, l'apprenti traducteur doit apprendre à procéder à une forme de dédoublement de sa personnalité; oublier son œuvre et la traiter comme celle d'une autre personne. Certaines erreurs ne se repèrent pas, même si elles devraient sauter aux yeux, car l'esprit colle sur les fausses interprétations. Dans certains cas, la conviction de la justesse de la première interprétation est telle que le traducteur, au lieu de procéder à une nouvelle interprétation en se détachant au maximum de son œuvre, considère qu'il a traduit un texte «mal rédigé». Ainsi, au lieu de vérifier sa première interprétation, il adapte ses choix qui suivent afin que son texte soit «cohérent», ce qui implique la production de contresens en chaîne.

Une activité mentale liée à la lecture et qui joue un rôle important dans toutes les étapes du processus traductionnel est celle de l'attention. Dans le cadre des recherches à effectuer, nous devons étudier la manière dont le traducteur gère ses ressources attentionnelles lors des différentes étapes de la traduction ou lors de la gestion de certains types de difficultés, car il semble que plusieurs types d'erreurs relèvent d'une mauvaise application de l'attention. De même, nous serons amenés à répondre à des questions telles que: de quelle manière l'attention influe-t-elle sur la fiabilité et les performances du traducteur, quels sont les effets de l'attention sur la charge mentale 
requise pour l'accomplissement de l'acte traductionnel, peut-on proposer des stratégies de régulation de l'attention en fonction du degré de difficulté traductionnelle, de la quantité du travail à effectuer, etc.

Une étape de l'acte traductionnel qui mérite également une étude particulière est celle de la reformulation du sens, car elle implique une forme singulière d'activité rédactionnelle. Le traducteur, bien qu'il soit contraint par le vouloir dire et le dit de l'auteur dont il traduit l'œuvre, doit élaborer sa propre stratégie rédactionnelle en tenant compte des paramètres communicationnels qui régissent l'acte traductionnel. La rédaction de la traduction peut être considérée comme un problème qui requiert une solution, comme une série de prises de décisions. Elle doit être également considérée comme objet d'une approche relevant à la fois de l'ergonomie et de la psychologie cognitive, dans la mesure où la rédaction de la traduction suppose une organisation particulière du «bureau» du traducteur de sorte qu'il puisse optimiser son travail au moindre «coût cognitif».

Un autre domaine vers lequel nous pensons que la recherche doit s'orienter est celui du fonctionnement du système mnésique des apprentis traducteurs. En acceptant l'approche d'Alan Baddeley concernant l'organisation et le fonctionnement du système mnésique, nous pouvons soutenir que l'essentiel du traitement des informations lors de l'acte traductionnel a lieu dans ce qu'il a appelé la «mémoire de travail». Il s'agit d'un système qui est chargé de traiter les informations issues du milieu extérieur dans le cadre du processus de la perception, tout en exploitant les ressources cognitives du sujet contenues dans sa mémoire à long terme. Lors de la traduction, la mémoire de travail devrait traiter les informations contenues dans le texte à traduire, les informations extralinguistiques qui régissent ce texte ainsi que les informations pertinentes contenues dans la mémoire à long terme du traducteur que ce dernier mobilise en vue d'accomplir sa tâche.

Ce processus complexe implique l'élaboration d'une représentation mentale de l'énoncé et son traitement en vue de servir de base pour la reformulation du sens en tenant compte des normes de la langue d'arrivée. L'étude de l'élaboration des représentations mentales et de son traitement lors de la traduction nous fournira des informations d'un grand intérêt car elle éclaircira le processus de la compréhension du sens et de sa reformulation.

Cette mémoire, bien qu'elle ne soit pas un système souple, permet d'envisager l'élaboration de stratégies plus efficaces du point de vue qualificatif en vue d'améliorer ses performances. L'expérience dans l'enseignement de la traduction nous montre que les étudiants, en fonction de leur niveau, de leur expérience, mais aussi de leur empan mnésique peuvent traiter un volume et une qualité d'informations différents. Pour ce faire, ils adoptent des stratégies de traitement différentes. Il suffit de donner à un étudiant de première année un texte contenant plusieurs points qui représentent des «difficultés » pour un apprenti traducteur pour constater qu'il peine énormément pour maîtriser l'ensemble des informations explicites ou implicites du texte qu'il a à traduire. Si, par exemple, nous lui donnons à traduire une phrase longue, contenant une ou deux subordonnées, une ou deux locutions non connues et une ou deux notions abstraites, sans lui offrir une aide extérieure, nous constaterons qu'une fois concentré sur l'interprétation du troisième ou du quatrième point «difficile», il reviendra au premier point car sa mémoire de travail aura besoin d'un soutien, d'un coup de pouce, pour réaliser le traitement de l'ensemble des informations. Il s'agit 
d'une «défaillance» relative de la mémoire de travail qui se corrige au fur et à mesure, essentiellement par un travail personnel. En fait, l'apprenti traducteur est appelé à traiter parallèlement plusieurs informations supposant des degrés de concentration différents. La concentration sur l'un de ces problèmes implique une difficulté pour garder en mémoire de travail des informations issues des traitements précédents. Pour y arriver, l'apprenti traducteur doit s'appliquer à accomplir parallèlement au moins deux tâches cognitives distinctes, à savoir la prospection du sens d'un fragment de l'énoncé tout en gardant en mémoire active, par des procédés mnémoniques particuliers, les informations issues des traitements ultérieurs.

Le traitement simultané et combiné de plusieurs informations est la source du phénomène de la surcharge mentale qui, à partir d'un certain moment, entrave ou empêche le traitement efficace des informations en mémoire de travail lors de la traduction. Le but des recherches dans ce domaine devrait consister à étudier ce phénomène et, en fonction des résultats, proposer aux apprentis traducteurs des exercices leur permettant de mieux gérer leurs ressources cognitives requises. Ces exercices viseront également à l'amélioration de leurs performances telles que la quantité d'informations traitées par unité de temps, la qualité du traitement effectué, la quantité et la qualité d'informations extraites de la mémoire à long terme, etc.

La référence à la charge mentale requise et à son rapport avec les «difficultés » lors du traitement des informations en traduction nous amène à la notion du degré de difficulté traductionnelle. Selon Eleni Sella et Fridériki Batsalia, dans leur approche linguistique de cette notion, le degré de difficulté traductionnelle d'une unité de traduction dépend du nombre de «déplacements » obligatoires des éléments morphosyntaxiques, sémantiques, lexicologiques et pragmatologiques du texte de départ, de leur position initiale à un autre point d'une échelle idéelle commune des systèmes de la langue de départ et de la langue d'arrivée. Bien qu'à priori cette approche soit linguistique, elles ajoutent à leur modèle une dimension qui relève plutôt des sciences cognitives que de la linguistique classique, à savoir un paramètre qui exprime le niveau d'interprétation requis. Elles en distinguent trois niveaux: les niveaux d'interprétation 0,1 et 2 .

Dans une approche cognitive, nous aurons tendance à formuler l'hypothèse que le degré de difficulté traductionnelle devrait dépendre en principe de la difficulté à élaborer la représentation mentale de l'énoncé, de la difficulté de son traitement et de la charge mentale exigée par le traducteur en vue de réaliser son œuvre. Cette charge ne devrait pas dépendre uniquement des informations explicites ou implicites contenues dans le texte, mais aussi des facteurs cognitifs, tels que les capacités de traitement de la mémoire de travail du traducteur, de son bagage cognitif, de son expérience en la matière, etc.

Un sujet qui peut être également étudié dans le cadre des études concernant la mémoire de travail en traduction écrite est celui du rôle des niveaux multiples de traitement des informations, mais aussi du rôle du traitement différé des informations lors de la traduction. Il s'agit du phénomène d'incubation, qui, certaines fois, permet au traducteur de saisir correctement l'intégralité du sens du texte qu'il traduit, non plus au moment du traitement conscient des informations qu'il a sous les yeux, mais à un moment différé. C'est ainsi que nous pourrions expliquer le phénomène de révision intégrale ou partielle d'une grande partie de leur traduction chez certains apprentis traducteurs. 
Nos investigations ne doivent pas se limiter au domaine de la mémoire de travail, mais elles doivent s'étendre également à la mémoire à long terme, et plus particulièrement à la mémoire déclarative. Ce qui nous intéresse particulièrement est la manière dont sont organisées et peuvent être exploitées les représentations sémantiques ou épisodiques susceptibles d'être traitées lors de l'acte traductionnel. Dans le cadre de ces recherches, nous pourrions même étudier si dans l'acte traductionnel existent des procédés cognitifs qui par l'exercice et la surformation atteignent un degré d'automatisme, ce qui pourrait impliquer un amoindrissement de la charge mentale requise pour effectuer une traduction. Si ceci s'avère véridique, nous pourrions proposer aux étudiants des exercices spécifiques leur permettant le développement de ces automatismes.

Si l'on tient compte des domaines de coopération des traductologues et des spécialistes en psychologie cognitive ainsi que des défis lancés, il ne serait pas sans fondement de prétendre que nous entrons dans une nouvelle phase de maturité de cette coopération. Les résultats attendus offriront aux enseignants de traduction des outils précieux pour enrichir leurs cours en vue de former des traducteurs plus fiables et plus performants.

\section{RÉFÉRENCES}

\section{Références en grec}

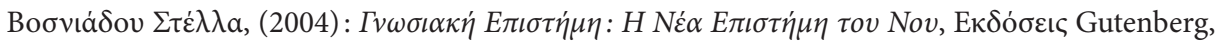
A $\theta$ ńva

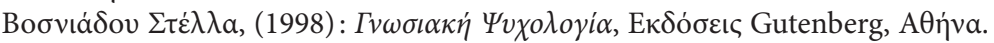

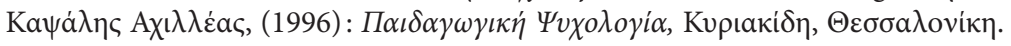

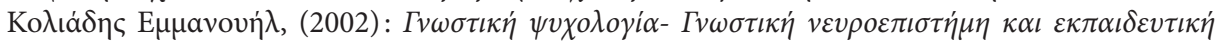
$\pi \rho \dot{\xi} \xi \eta, \mathrm{A} \theta \dot{\eta} v \alpha$.

M

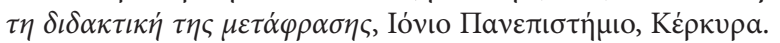

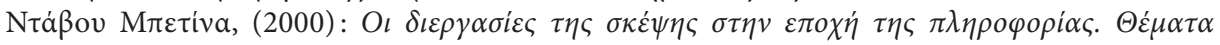

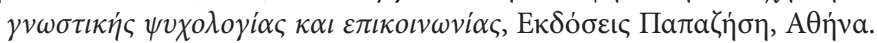

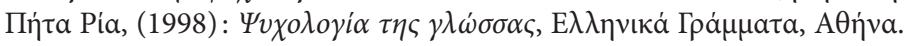

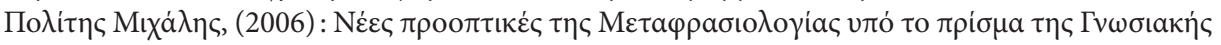

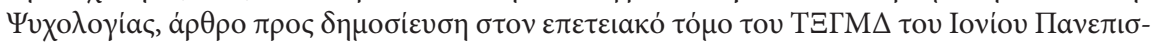

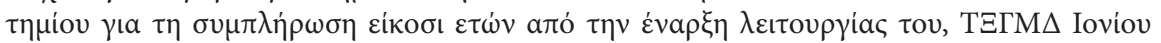

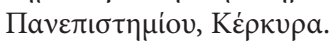

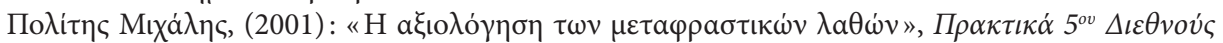

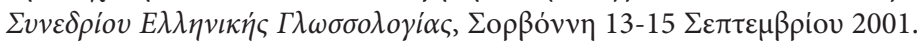

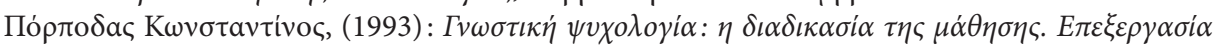

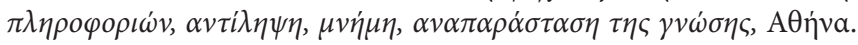

\section{Références en français}

CAdet, B. (1998): Psychologie cognitive, Paris, In Press.

Coirier, P., Gaonac'h, D. et J.-M. Passerault (1996): Psycholinguistique textuelle. Approche cognitive de la compréhension et de la production des textes, Paris, Armand Colin.

Dancette, J. (1995): Parcours de traduction, Lille, Presses Universitaires de Lille.

Delisle, J., Lee-Jahnke, H. et M. C. Cormier (1999): Terminologie de la traduction, Amsterdam/ Philadelphia, John Benjamins Publishing Company.

Delisle, J. (1984): L'analyse du discours comme méthode de traduction, Ottawa, Éditions de l'Université d'Ottawa.

Durieux, C. (2005): «La traduction: illustration d'un processus complexe», La complexité: ses formes, ses traitements, ses effets, Caen, Cahiers de la MRSH de l'Université de Caen. 
Durieux, C. (2004): "Approche psycholinguistique de la traduction», Psycholinguistics: A Multidisciplinary Science, Paris, Europia.

Durieux, C. (1995): Apprendre à traduire, Paris, Maison du Dictionnaire.

Durieux, C. (1990): «La recherche documentaire en traduction technique: conditions nécessaires et suffisantes», Meta 35-4, Les Presses de l'Université de Montréal.

Durieux, C. (1988): Fondement didactique de la traduction technique, Paris, Didier Érudition.

ESIT et Groupe d'études sur le langage de l'Université Paris XII (1981): Actes du Colloque Compréhension du langage, Créteil 25-27 septembre 1980, Paris, Didier Érudition.

Kosma, A. (2005): Le fonctionnement spécifique de la mémoire de travail en traduction, Caen (mémoire en vue de l'obtention du master conjoint «Sciences de la traduction: traductologie et science cognitive»).

Larose, R. (1998): "Méthodologie de l'évaluation des traductions», Meta 43-2, Les Presses de l'Université de Montréal.

Lederer, M. (1994): La traduction aujourd'hui, Paris, Hachette.

Lemaire, P. (1999): Psychologie cognitive, Bruxelles, De Boeck.

Le Ny, J.-F. (1989): Science cognitive et compréhension du langage, Paris, Presses Universitaires de France.

Matlin, M. (2001): La cognition, Bruxelles, De Boeck.

Mialet, J.-P. (1999): L'attention, Paris, Presses Universitaires de France, coll. "Que saisje?»(traduit en grec par les étudiantes du DLETI de l'Université ionienne Olga Daniilidou et Maria Hatzissava sous forme de mémoire en vue d'obtenir leur maîtrise).

Pergnier, M. (1980): Les fondements sociolinguistiques de la traduction, Paris, Libraire Honoré Champion.

Politis, M. (1999): «Approche théorique des erreurs en traduction spécialisée», Colloque sur la traduction, Athènes, Université d'Athènes.

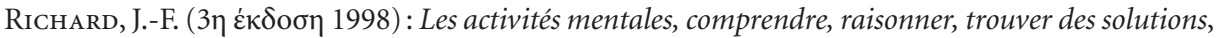
Paris, Armand Colin.

Seleskovitch, D. et M. Lederer (1981) : «Comprendre le langage. Le point de vue des linguistes, le point de vue des traducteurs interprètes, le point de vue des neuropsychologues ", in Actes du colloque Comprendre le langage, Paris, Didier Érudition.

Seleskovitch, D. et M. Lederer (1986): Interpéter pour traduire, Paris, Didier Érudition.

Seleskovitch, D. (1975): Langage, langue et mémoire, Paris, Minard.

VANDAele, S. et L. Lubin (2005), «Approche cognitive de la traduction dans les langues de spécialité: vers une systématisation de la description de la conceptualisation métaphorique», Meta 50-2, Les Presses de l’Université de Montréal.

\section{Références en anglais}

Baddeley, A. (1986): Working memory, Oxford, Oxford University Press.

BADDELEY, A. (1992): "Working Memory: the interface between memory and cognition", Journal of Cognitive Neuroscience 4, p. 281-288.

Baddeley, A. (2000): “The Episodic Buffer: A New Component of Working Memory?", Trends in Cognitive Sciences 4, p. 417-423.

BAdDeley, A. (2003): "Working Memory and Language: an Overview", Journal of Communication Disorders 36, p.189-208.

BAdDeley, A. (2003): "Working Memory: Looking Back and Looking Forward", Nature Reviews / Neuroscience 4, p. 829-839.

Kussmaul, P. (1995): Training the Translator, Amsterdam/Philadelphia, John Benjamins.

SÉGuinot, C. (1990): "Interpreting Errors in Translation", Meta 35-1, Les Presses de l'Université de Montréal.

WiLLs, W. (1996): Knowledge and skills in translator behaviour, Amsterdam/Philadelphia, John Benjamins. 